\title{
Thermodynamic and Conformational Study of Side On/Side End Fixed Liquid Crystalline Copolyacrylates
}

\author{
S. Lecommandoux $\left({ }^{1}\right)$, L. Noirez $\left({ }^{2}\right)$, M.F. Achard $\left({ }^{1}\right)$ and F. Hardouin $\left({ }^{1, *}\right)$ \\ ( ${ }^{1}$ Centre de Recherche Paul Pascal, Université Bordeaux I, avenue A. Schweitzer, 33600 Pessac, \\ France \\ $\left({ }^{2}\right)$ Laboratoire Léon Brillouin (CEA-CNRS), CE Saclay, 91191 Gif-sur-Yvette, France
}

(Received 18 March 1996, received in final form and accepted 3 June 1996)

PACS.61.30.-v - Liquid crystals

PACS.62.20.- $\mathrm{x}$ - Mechanical properties of solids

PACS.66.20.+d - Viscosity of liquids; diffusive momentum transport

\begin{abstract}
Small angle neutron scattering experiments were carried out on liquid crystalline copolyacrylates with various proportions of mesogenic groups attached laterally ("side-on fixed") and terminally ("side-end fixed") to the polymer backbone. The influence of the competition between these two different kinds of fixation on the chain conformation is for the first time evidenced. Side-on fixation prevails upon side-end one from thermodynamical, structural and conformational points of view. A relation between the order of the nematic phase and the anisotropy of the backbone is clearly evidenced.
\end{abstract}

Résumé. - Des copolyacrylates à chaînes latérales comprenant à la fois des groupements mésomorphes attachés latéralement (fixation en haltère) et longitudinalement (fixation en peigne) au squelette polymère sont étudiés par diffusion de neutrons aux petits angles. L'influence de la compétition entre ces deux types d'accrochage sur la conformation de la chaîne polymère est mise en évidence pour la première fois. La fixation en haltère a une influence prédominante sur les propriétés thermodynamiques, structurales et conformationnelles des copolymères. Une relation directe entre l'ordre de la phase nématique et l'anisotropie de la chaîne polymère est établie.

\section{Introduction}

Side-on fixed liquid crystalline polymers, in which the mesogenic groups are laterally branched to the backbone, exhibit specific characteristics by comparison with the conventional side-end fixed LCP. In spite of some examples of smectic phases in this kind of LCP [1-5], the lateral arrangement generally flavors the nematic state [6-16]. In contrast, the side-end fixed polymers are predominantly smectogenic [17]. Moreover, earlier studies by Small Angle Neutron Scattering (SANS) emphasized the difference of the backbone conformation shape between side-on and side-end fixed LCP.

Laterally attached polymers form a "jacketed structure" at the scale of the chain dimension in the nematic phase: the backbone is strongly stretched in the average direction imposed by its

$\left(^{*}\right)$ Author for correspondence (e-mail: hardouin@crpp.u-bordeaux.fr)

(C) Les Éditions de Physique 1996 
mesogenic groups [18-25]. The influence of the chemical architecture has been studied during these last years. It has been demonstrated that the anisotropy of the backbone conformation is reduced when the spacer length increases, implying a smaller jacketed effect [21,24]. Moreover, an increase of the length of the aliphatic extremities reduces the force of this jacketed effect [24]. Finally, the same evolution is observed decreasing the amount of mesogenic groups by incorporating flexible non-mesogenic units [20,23,24].

Qualitatively speaking, terminally attached LCP exhibit an oblate shape of the backbone conformation in the smectic A phases, traducing a confinement of the chains between the mesogenic layers [26-28]. This stimulating situation made us interested in understanding more precisely these opposed interactions and in turn, their contribution in a same basic system. That is why we aim at studying for the first time the backbone conformation of statistical copolymer systems including side-on and side-end attached mesogenic units. Indeed, this is the best way to combine the physical properties of the two kinds of homopolymer avoiding the problem of immiscibility $[29,30]$. Note that the corresponding homopolyacrylates have been extensively studied exhibiting fundamental differences in their mesomorphic and conformational behaviours $[21,31]$.

\section{Synthesis and Characterization of the LC Copolyacrylates}

2.1. SYNTHESIS. - The polymers and copolymers have the following general formula:

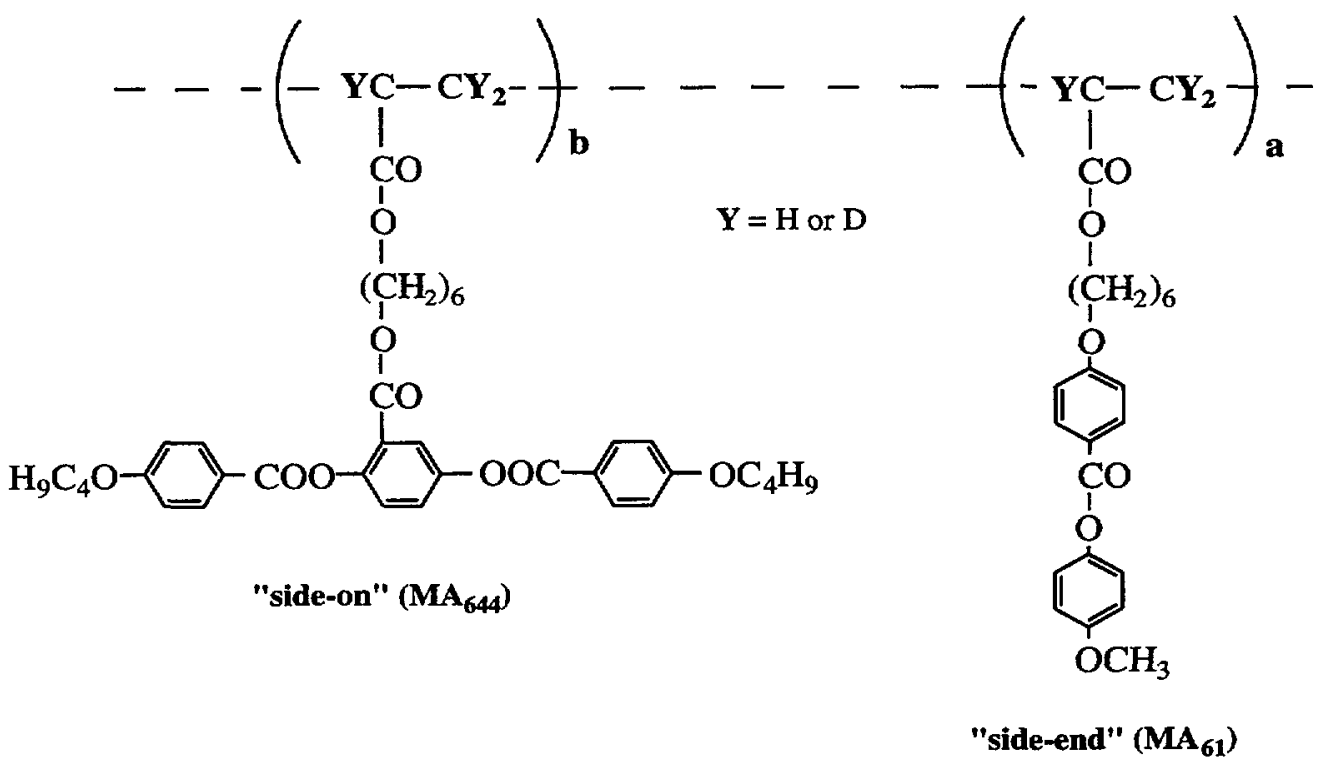

Each copolymer is obtained from a mixture of the side-on fixed $\left(\mathrm{MA}_{644}\right)$ and side-end fixed $\left(\mathrm{MA}_{61}\right)$ monomers: for example to prepare the copolyacrylate with $20 \%$ of $\mathrm{MA}_{61}$, the monomers $\mathrm{MA}_{61}(100.2 \mathrm{mg}, 0.27 \mathrm{mmol})$ and $\mathrm{MA}_{644}(712.8 \mathrm{mg}, 1.08 \mathrm{mmol})$ are added to $1.35 \mathrm{~cm}^{3}$ of dry dioxane (monomer concentration: $10^{-3} \mathrm{~mol} \mathrm{~cm}{ }^{-3}$ ) with $1.5 \% \mathrm{~mol}$ of azobis(isobutyronitrile) $(3.3 \mathrm{mg})$. The mixture was stirred and heated at $70^{\circ} \mathrm{C}$, with the exclusion of moisture (under nitrogen atmosphere) during 8 hours. The solvent was removed, then the polymer was dissolved in dry tetrahydrofuran and repeatedly precipitated with cold methanol. The polymer was then filtered and dried at $80^{\circ} \mathrm{C}$ under vaccuo for 8 hours. We used the same procedure for each 
copolymer, controlling precisely the mass of AIBN and the volume of dioxane. It results the same average molecular weights.

For the sake of simplicity, the copolymers are named copoPA- $X$ (H or D) with $X=a /(a+b)$ the percentage of the monomer $\mathrm{MA}_{61}$ and $(1-X)$ the percentage of $\mathrm{MA}_{644} ; \mathrm{H}$ for the completely hydrogenated polymer and $\mathrm{D}$ for the partially deuterated one.

2.2. Characterization of the Copolymers. - The molecular weights and the polydispersity of the polyacrylates samples were measured by size exclusion chromatography (SEC) from polymer solutions in toluene versus polystyrene standards. The phase transition temperatures were determined by differential scanning calorimetry (DSC) and optical measurements. These results are summarized in Table $\mathrm{I}$.

Table I. - Characterization of the polymers by SEC and DSC. $M_{\mathrm{w}}=$ weight average molecular weight. $I=M_{\mathrm{w}} / M_{n}=$ polydispersity index. $D P_{n}=$ average degree of polymerization in number. $g=$ glassy state ( ${ }^{*}$ from DSC measurements with a speed rate of $-10{ }^{\circ} \mathrm{C} \mathrm{min}^{-1}$ ). $N=$ nematic phase. $S_{\mathrm{A}}=$ smectic $A$ phase. $I=$ isotropic liquid phase. $\Delta H_{\mathrm{IN}}=$ enthalpy change at the IN transition ( $J \mathrm{um}^{-1}$ Joule per unity of monomer) $\Delta S_{\mathrm{IN}}=$ entropy change at the IN transition ( $J \mathrm{~K}^{-1} \mathrm{um}^{-1}$ Joule per Kelvin per monomeric unit).

\begin{tabular}{|l|c|c|c|c|c|c|c|}
\hline polyacrylates & & $M_{\mathrm{w}}$ & $I$ & $\mathrm{DP}$ & transition temperatures & $\begin{array}{c}\Delta H_{\mathrm{IN}} \\
\left(\frac{\mathrm{J}}{\mathrm{um}}\right)\end{array}$ & $\begin{array}{c}\Delta S_{\mathrm{IN}} \\
\left(\frac{\mathrm{J}}{\mathrm{K} \mathrm{um}}\right)\end{array}$ \\
\hline PA $_{644}$ & $\mathrm{H}$ & 34000 & 1.8 & 30 & $\mathrm{~g}-28^{*}-\mathrm{N}-114-\mathrm{I}$ & 1509 & 3.9 \\
\hline from Ref. [9] & $\mathrm{D}$ & 99000 & 1.7 & 85 & $\mathrm{~g}-28^{*}-\mathrm{N}-114-\mathrm{I}$ & 1509 & 3.9 \\
\hline copoPA-0.20 & $\mathrm{H}$ & 50000 & $\cong 2$ & 41 & $\mathrm{~g}-30^{*}-\mathrm{N}-109-\mathrm{I}$ & 917 & 2.4 \\
\hline & $\mathrm{D}$ & 100000 & $\cong 2$ & 82 & $\mathrm{~g}-32^{*}-\mathrm{N}-107-\mathrm{I}$ & 874 & 2.3 \\
\hline copoPA-0.45 & $\mathrm{H}$ & 55000 & $\cong 2$ & 52 & $\mathrm{~g}-32^{*}-\mathrm{N}-109-\mathrm{I}$ & 649 & 1.7 \\
\hline & $\mathrm{D}$ & 75000 & $\cong 2$ & 70 & $\mathrm{~g}-31^{*}-\mathrm{N}-109-\mathrm{I}$ & 649 & 1.7 \\
\hline copoPA-0.80 & $\mathrm{H}$ & 50000 & $\cong 2$ & 58 & $\mathrm{~g}-28^{*}-\mathrm{N}-113-\mathrm{I}$ & 502 & 1.3 \\
\hline & $\mathrm{D}$ & 60000 & $\cong 2$ & 69 & $\mathrm{~g}-29^{*}-\mathrm{N}-113-\mathrm{I}$ & 502 & 1.3 \\
\hline PA $_{61}$ & $\mathrm{H}$ & 27000 & 2.6 & 30 & $\mathrm{~g}-22^{*}-\mathrm{S}_{\mathrm{A}}-88-\mathrm{N}-116-\mathrm{I}$ & 490 & 1.3 \\
\hline from Ref. [14] & $\mathrm{D}$ & 41000 & 2.0 & 55 & $\mathrm{~g}-23^{*}-\mathrm{S}_{\mathrm{A}}-88-\mathrm{N}-114-\mathrm{I}$ & 512 & 1.3 \\
\hline
\end{tabular}

The evolution of the transition temperatures can be represented in a pseudo phase diagram (Fig. 1). We note the strong influence of the "side-on" fixation on the mesomorphic properties: the nematic state induced by the side-on fixation exists up to $80 \%$ of side-end fixed mesogenic groups and the smectic A phase relative to the side-end fixed homopolymer is strongly destabilized by the insertion of a small amount of side-on mesogens. A similar evolution dominated by a strong side-on nematic regime has been already reported in side-on/side-end fixed copolysiloxanes systems $[29,30]$. 


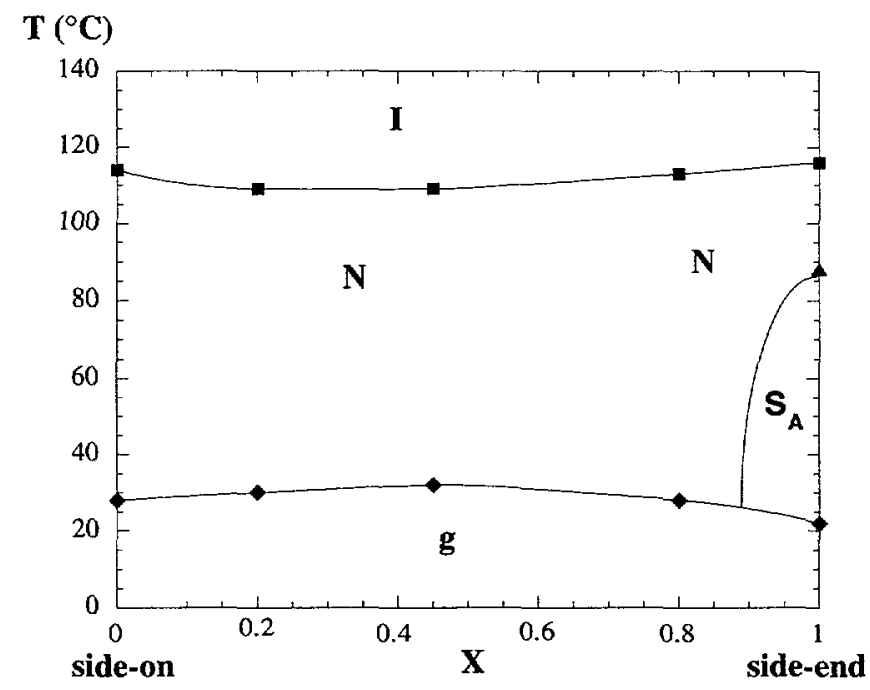

Fig. 1. - Pseudo phase diagram of the side-on/side-end fixed copolymer system copoPA- $X$ where $X$ is the percentage of the side-end fixed mesogen. $g=$ glassy state, $N=$ nematic phase, $S_{A}=$ smectic A phase, $\mathrm{I}=$ isotropic liquid phase.

Moreover, the IN transition enthalpy $\left(\Delta H_{\mathrm{IN}}\right)$ and entropy $\left(\Delta S_{\mathrm{IN}}\right)$ strongly decreases from the side-on homopolymer to copolymers (Tab. I). This evolution traduces the weakening of the chain-mesogen and mesogen-mesogen couplings.

2.3. Local Order in the Nematic Phase by X-Ray Diffraction. - X-ray diffraction experiments were performed on oriented samples to characterize the local order in the nematic phase. Orientation of the samples was achieved by cooling down from the isotropic phase in a 4.2 Tesla magnetic field and X-ray patterns of the magnetically oriented polymers were recorded at room temperature in their nematic or glassy state.

As previously reported $[3,32,33]$ the patterns of side-on homopolymers exhibit several diffuse elements indicating different kinds of short range order: in addition to the usual wide angle diffuse rings located on the equator associated with the disordered lateral arrangement of the mesogenic groups oriented along the magnetic field, four diffuse spots located at small angles off the meridian are commonly observed. They originate from local correlations of the aliphatic parts of the mesogenic units [3] and appear characteristic of the laterally branched architecture as discussed elsewhere [34].

It is worthnoting that the patterns corresponding to copolymers with $X=0.2$ and $X=0.45$ appear very similar to the pattern of the homopolymer $\mathrm{PA}_{644}$ and present a local order typically representative of the side-on fixation.

At the opposite, the four diffuse spots are no longer observed in the pattern of the copolymer corresponding to a majority of the "side-end" mesogens $(X=0.8)$ : only remain diffuse lines perpendicular to the director which indicate that the mesogenic groups tend to align themselves in strings over a short range. Such diffuse elements are observed as well for the side-on homopolymer $[3,32]$ as for the side-end fixed one [35] and in this sense is not characteristic of one type of fixation.

Thus from a structural point of view, one can note that the influence of the lateral fixation prevails at least up to equal proportion of each kind of mesogenic units. 


\section{Small Angle Neutron Scattering Experiments (SANS)}

The sample used for the SANS experiments is a homogeneous polymer pseudo-mixture in bulk consisting of $\mathrm{H}$ and $\mathrm{D}$ liquid crystal polyacrylates with very similar $\mathrm{DP}_{n}$ values at 50/50 weight ratio. Each mixture is prepared by dissolving the two copolyacrylates $(\mathrm{H}$ and $\mathrm{D})$ in tetrahydrofuran and then evaporating the solvent. The powder obtained is dried under vacuum at $80^{\circ} \mathrm{C}$ and then placed in a quartz cell which is put in an oven. The SANS experiments were performed on the PAXY spectrometer at the LLB (Orphée reactor, Saclay) equipped with an $X Y$ position sensitive multidetector using a well-known procedure described elsewhere [36]. An oriented nematic phase is obtained in situ by cooling the sample from the isotropic phase under an external magnetic field of 1.4 Tesla: the orientation of the mesogens is then achieved in the horizontal plane and perpendicularly to the neutron beam.

The wavelength used for the scattering experiments was $\lambda=10 \AA$ and the sample - multidetector $\left(128 \times 128\right.$ cells of $\left.5 \times 5 \mathrm{~mm}^{2}\right)$ distance was 2 meters. The scattering vector range explored ( $q=4 \pi \sin \theta / \lambda \cong 4 \pi \theta / \lambda$, where $2 \theta$ is the scattering angle assumed small) is then:

$$
7 \times 10^{-3} \AA^{-1} \leq q \leq 7 \times 10^{-2} \AA^{-1}
$$

The scattering patterns of the oriented samples present an anisotropy (Fig. 2a). The intensities recorded in the detector cells of the same $q$ modulus are regrouped by rectangular sectors of $10 \times 128$ cells in the parallel direction to the magnetic field and $8 \times 128$ cells in the perpendicular one. This gives the primary scattering intensities $I\left(q_{\|}\right)$and $I\left(q_{\perp}\right)$ respectively. The linear dependence of the inverse of the intensity scattered by the backbones $I^{-1}(q)$ versus $q^{2}$ is obtained after the data treatment (Fig. 2b). In this range, which is the enlarged Guinier domain $(q R \leq 2)$, we can write:

$$
\begin{aligned}
& I^{-1}\left(q_{\|}\right)=I^{-1}(0)\left(1+q_{\|}^{2} R_{\|}^{2}\right) \\
& I^{-1}\left(q_{\perp}\right)=I^{-1}(0)\left(1+q_{\perp}^{2} R_{\perp}^{2}\right)
\end{aligned}
$$

where $R_{\|}$and $R_{\perp}$ are the apparent components of the radius of gyration of the polymer backbone in the directions parallel and perpendicular to the magnetic field.

The difference in molecular weight between the labelled and the unlabelled polymer has been taken into account using a law derivative from the Guinier approximation [34]. $R_{\text {iapp }}$ ( $\mathrm{i}=\|$ or 1 ), used above, is an apparent radius of gyration in the direction $q_{i}$ which is a function of both molecular weights of the two polymers. It is related to the size $R_{\mathrm{iD}}$ of the labelled chains by $[26,37]$ :

$$
R_{\mathrm{iapp}}^{2}=R_{\mathrm{iD}}^{2}\left(\frac{1+\phi_{\mathrm{D}}}{\phi_{\mathrm{H}}+M_{\mathrm{D}} /\left(M_{\mathrm{H}}-M_{\mathrm{D}}\right)}\right)
$$

where $\phi_{\mathrm{H}}, \phi_{\mathrm{D}}$, are the volume fractions, $M_{\mathrm{H}}, M_{\mathrm{D}}$ are the molecular weights of the hydrogenated chains and the deuterated chains respectively.

This relation, at first established for isotropic systems [37], is valid for our experiments. This scattering law assumes that no specific interaction occurs between labelled and unlabelled polymers; this is actually experimentally checked by following the extrapolated intensity value at the origin for each temperature [38]. Since this value does not change, we conclude that there is no isotopic segregation in our copolymer melts.

We have

$$
\phi_{\mathrm{D}}=\phi_{\mathrm{H}}=0.5
$$

Then

$$
R_{\mathrm{iD}}^{2}=0.5 R_{\text {iapp }}^{2}\left[1+\left(\frac{M_{\mathrm{D}}}{M_{\mathrm{H}}}\right)\right]
$$




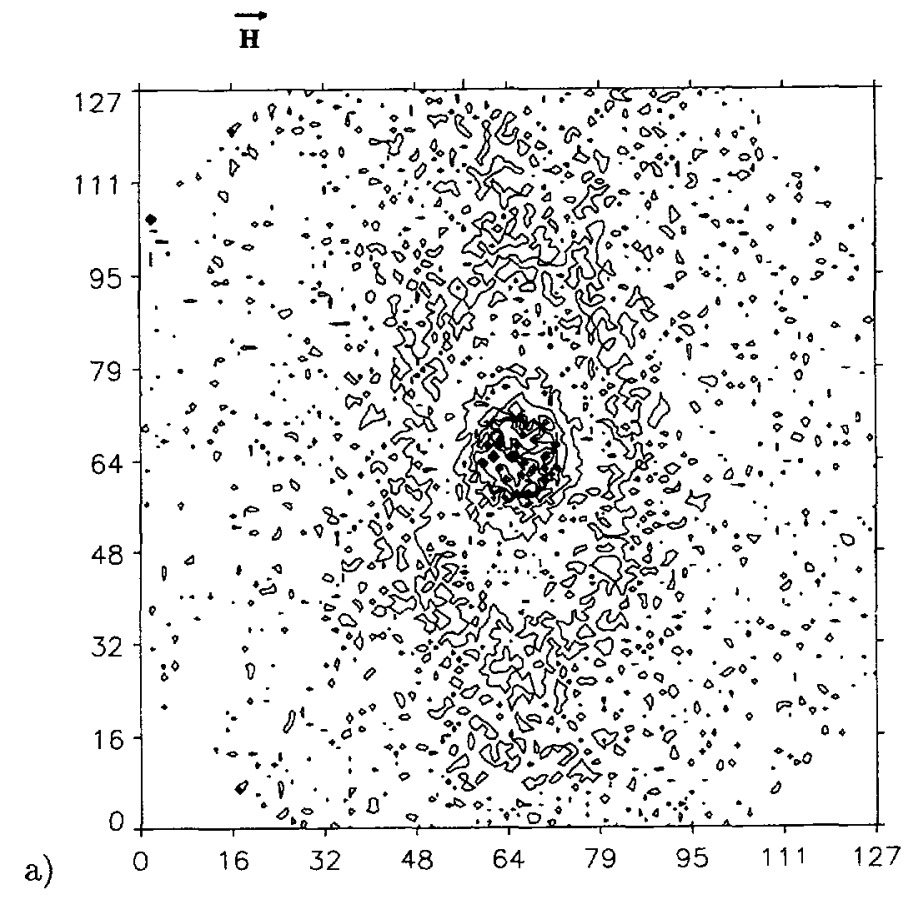

\section{$\mathbf{I}^{-1}(\mathbf{a . u .})$}

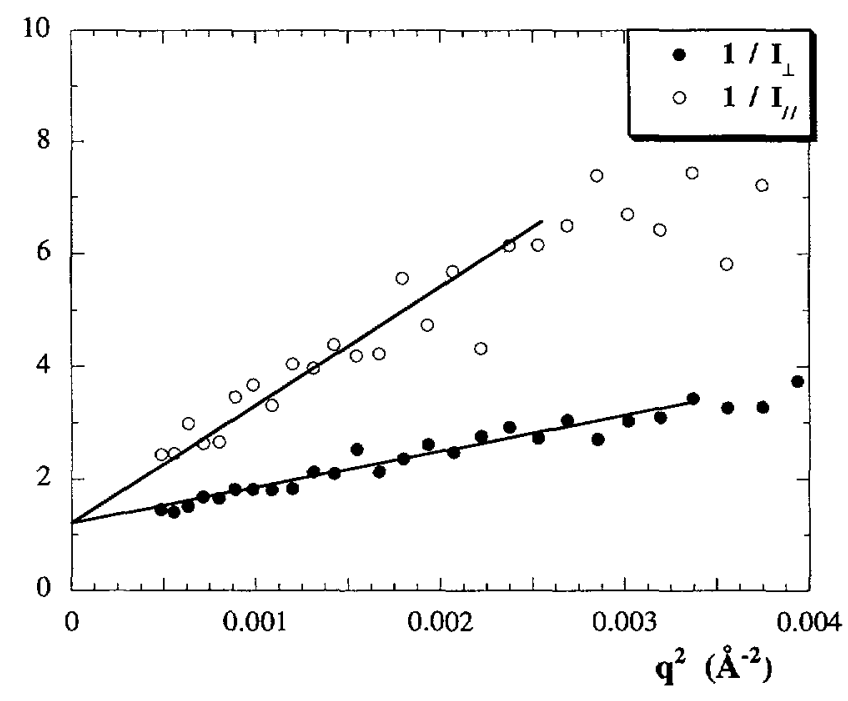

Fig. 2. - a) Transverse section of intensity scattered by the polymer backbone of the copoPA- 0.45 in nematic phase $\left(T=65^{\circ} \mathrm{C}\right)$. The magnetic field is horizontal. b) Inverse of the SANS intensity plotted in arbitrary units as a function of $q^{2}$ for the copoPA- 0.45 in nematic phase $\left(T=65^{\circ} \mathrm{C}\right)$. For both $\|$ and $\perp$ directions, the lines are obtained from a least square method in the enlarged Guinier domain $(q R \leq 2)$. 
Therefore we obtain:

$$
\begin{array}{rll} 
& R_{\mathrm{iD}}=1.22 R_{\text {iapp }} & \text { for copoPA }-0.2 \\
& R_{\mathrm{iD}}=1.09 R_{\text {iapp }} & \text { for copoPA }-0.45 \\
\text { and } & R_{\mathrm{iD}}=1.05 R_{\mathrm{i} \text { app }} & \text { for copoPA }-0.8
\end{array}
$$

These corrections are very slight and do not change neither the scattering shape, nor the eventual anisotropy of the backbone.

From the representation of $I^{-1}(q)$ versus $q^{2}$ at different temperatures for each copolymer, we deduce the polymer dimensions $R_{\|}$and $R_{\perp}$. After corrections, the thermal dependence of these dimensions are reported in Figures $3 a-c$. The error is estimated about $5 \%$.

\section{Results and Discussion}

Let us first recall the results previously obtained for the homopolymers $\mathrm{PA}_{644}$ and $\mathrm{PA}_{61}$. For the side-on fixed homopolymer $\mathrm{PA}_{644}$ a prolate anisotropy of the backbone has been detected in the nematic phase indicating a strong nematic jacketed effect $\left(R_{\|} / R_{\perp}=4.5[21]\right)$. For the side-end fixed homopolymer $\mathrm{PA}_{61}$, the backbone conformation is clearly oblate in the smectic A phase. Nevertheless for this latter compound, the situation in the nematic phase appears more complex: just below the isotropic-nematic transition the backbone shows a faint prolate anisotropy (within the error bars) and at lower temperature it presents a weak oblate shape $\left(R_{\|} / R_{\perp}=0.6\right)$ traducing the existence of a consequent pretransitional effect of the $\mathrm{S}_{\mathrm{A}}$ order [31].

In the case of the copolymers under study, a prolate conformation of the backbone with respect to the nematic director is clearly evidenced for the three compositions (Figs. 3a-c). Decreasing temperature from the isotropic state to the nematic phase, the backbone size in the direction perpendicular to the director decreases down to $R_{\perp}=25 \AA$ for copoPA-0.2 and copoPA- 0.45 and to down to about $42 \AA$ for copoPA-0.8. At the same time, the polymer size in the direction parallel to the director increases up to $R_{\|}=70 \AA$ for copoPA-0.2, $R_{\|}=50 \AA$ for copoPA-0.45 and $54 \AA$ for copoPA-0.8. The radii of gyration $R_{\|}$and $R_{\perp}$ reach these saturated values at about 30 degrees below the isotropic-nematic transition.

As shown in Figure 4, the prolate shape observed for side-on fixed homopolymer is kept even in the case of a copolymer with a majority of side-end fixed mesogenic groups $(X=0.8)$. This point underlines the peculiar influence of the side-on fixation which forces the polymer to adopt a prolate anisotropy.

One can also remark in the Figure 4, that the anisotropy of conformation is in a first step more perturbated by the introduction of a small proportion of side-end mesogens: $R_{\|} / R_{\perp}$ decreases from 4.5 to 2.9 adding only $20 \%$ of side-end mesogens. For higher percentages of side-end mesogens, a quasi linear decrease of $R_{\|} / R_{\perp}$ is observed, characterizing the regular change from a prolate shape to a weakly oblate one.

It is obvious to say that in liquid crystalline polymers, the chain conformation is greatly influenced by the mesomorphic order. Here we try to compare the anisotropy of the polymer backbone $R_{\|} / R_{\perp}$, and the order of the nematic phase through the entropy change $\Delta S_{I N}$. The saturated values of the radii of gyration $R_{\|}$and $R_{\perp}$ are obtained at low temperature in the nematic phase while the entropy change $\Delta S_{\mathrm{IN}}$ corresponds to the isotropic-nematic transition. Nevertheless the Figure 5 , which represents $\left(R_{\|} / R_{\perp}\right) /\left(R_{\|} / R_{\perp}\right)_{\text {saturated }}$ versus $\left(T-T_{\mathrm{IN}}\right) / T_{\mathrm{IN}}$ outlines that all the polymers under study show in reduced coordinates the same thermal evolution of the backbone anisotropy. In this sense it is justified to compare the entropy variation $\Delta S_{\mathrm{IN}}$ and the saturated values of the anisotropy of the backbone conformation $\left(R_{\|} / R_{\perp}\right)_{\text {saturated }}$. Excepted for the side-end fixed homopolymer, a linear dependence of $\left(R_{\|} / R_{\perp}\right)_{\text {saturated }}$ versus 
$\mathbf{R}(\mathbf{\AA})$

a)

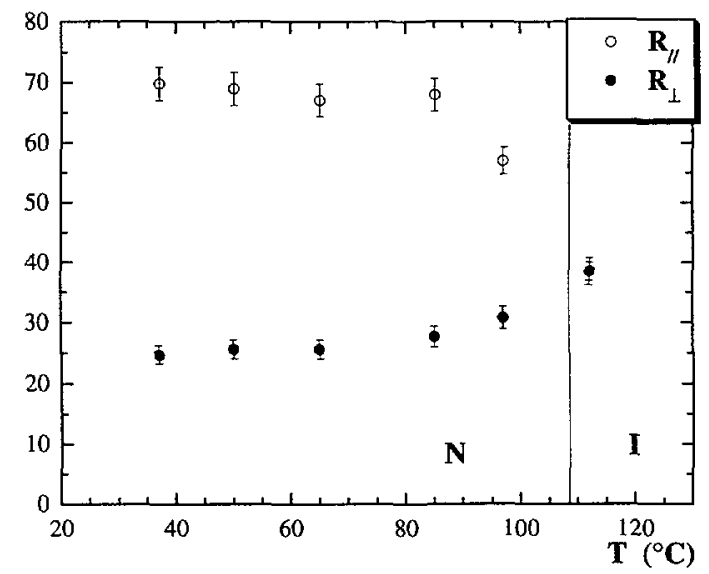

\section{$\mathbf{R}(\AA)$}

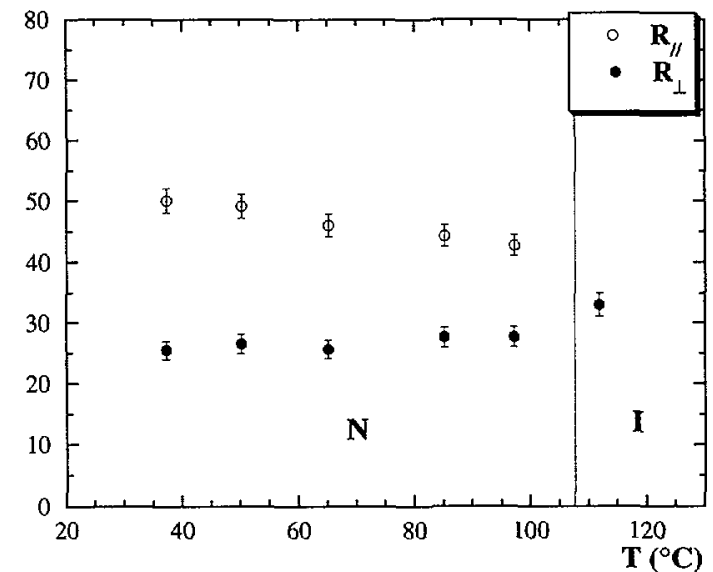

b)

$\mathbf{R}(\AA ̊)$

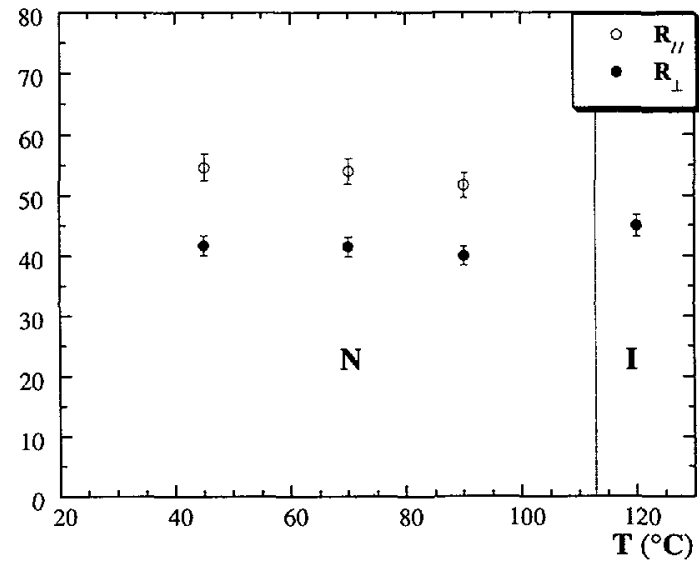

Fig. 3. - Thermal evolution in the nematic phase of the radii of gyration $R_{\|}$and $R_{\perp}$ of the polymer backbone (the index $\|$ means in the direction parallel to the nematic director and $\perp$ in the direction perpendicular): a) for copoPA-0.2. b) for copoPA-0.45. c) for copoPA-0.8. 


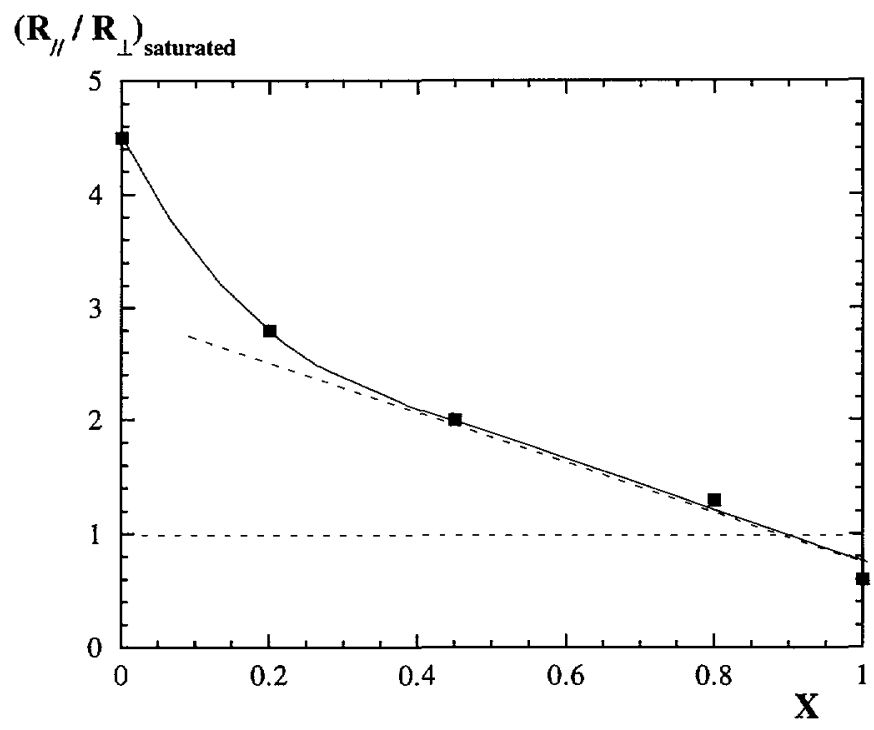

Fig. 4. - Evolution of the anisotropic ratio $\left(R_{\|} / R_{\perp}\right)_{\text {saturated }}$ as a function of the percentage $X$ of side-end fixed mesogens in copolymers. (The lines are just a guide for eyes).

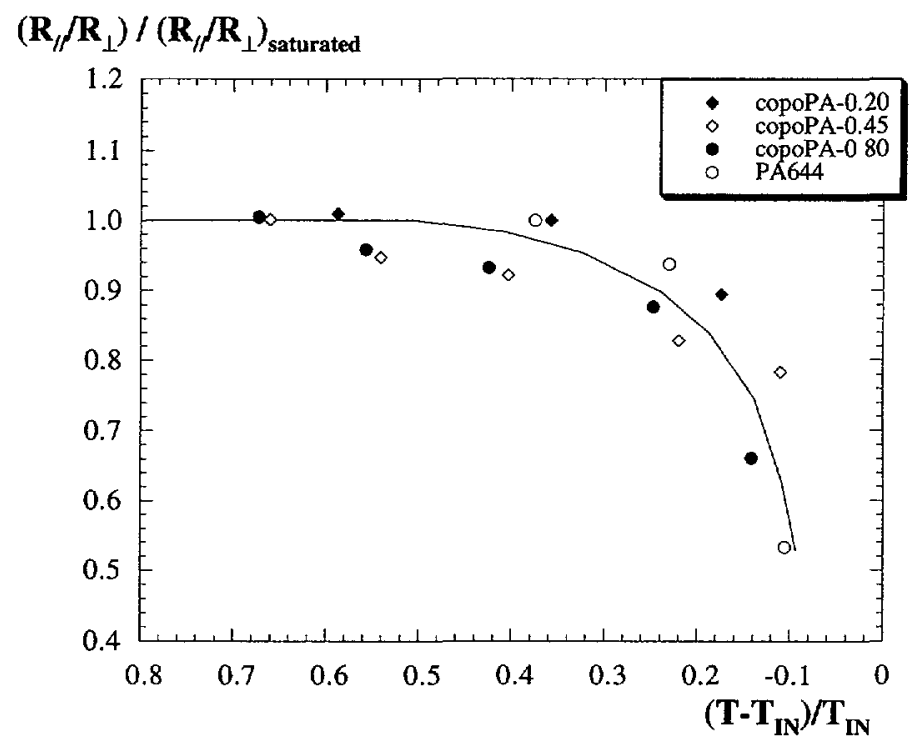

Fig. 5. - Evolution of the normalized anisotropic ratio $\left(R_{\|} / R_{\perp}\right) /\left(R_{\|} / R_{\perp}\right)_{\text {saturated }}$ versus the normalized temperature $\left(T-T_{\mathrm{IN}}\right) / T_{\mathrm{IN}}$. (The line is just a guide for eyes).

the isotropic-nematic entropy change $\Delta S_{\mathrm{IN}}$ is depicted in Figure 6. Thus, the anisotropy of the backbone conformation is directly connected to the nematic order in the lack of smectic $A$ fluctuations (i.e. in the prolate regime). As a consequence, the side-on homopolymer $\mathrm{PA}_{644}$ exhibits a significant entropy change $\Delta S_{\text {IN }}$ which can be interpreted as strong mesogen-chain and mesogen-mesogen interactions in the nematic phase leading to a stretched backbone with 


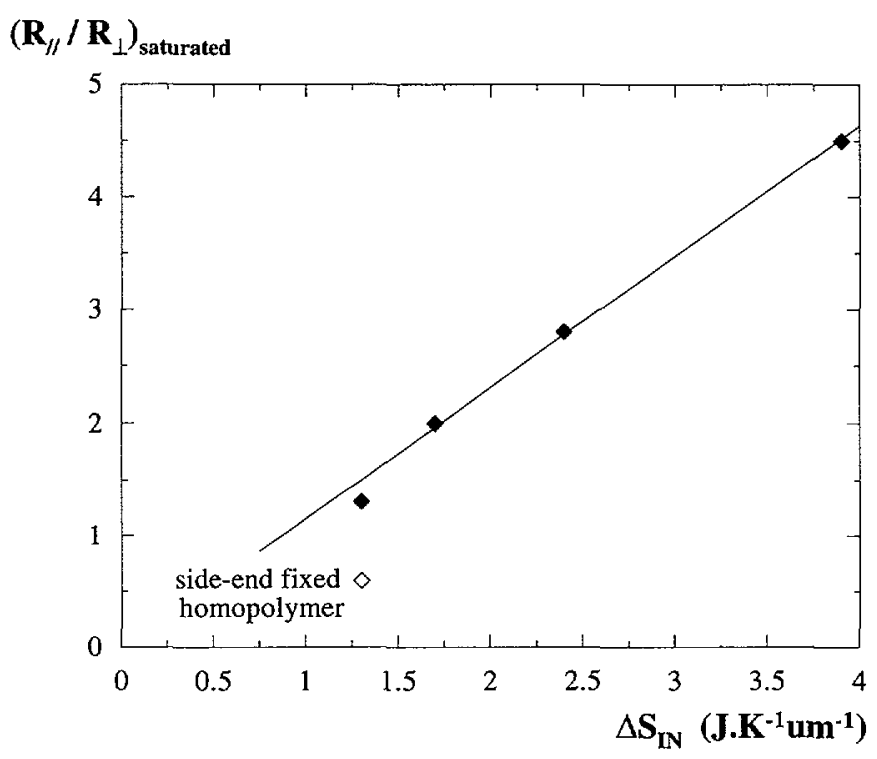

Fig. 6. - Evolution of the $\left(R_{\|} / R_{\perp}\right)_{\text {saturated }}$ versus the isotropic-nematic transition entropy $\Delta S_{\mathrm{IN}}$ (in Joules per Kelvin per monomeric unit).

a pronounced anisotropy of conformation. The introduction of "side-end" fixed mesogens unfavors this supra nematic ordering decreasing the interactions: the jacketed effect is weakened and as a result the participation of the backbone to the nematic order considerably decreases.

To sum up, these data clearly demonstrate that the diminution of the backbone anisotropy is directly related to a decrease of the order of the nematic phase and give evidence for the competition between the entropy maximization tendency of the polymer chain and the energy minimization of the nematic state. In addition, these evolutions are in good agreement with the Wang and Warner considerations on side-chains polymers [39]. By using a mean field model which combines a Maier-Saupe theory of conventional liquid crystals (where $\mathrm{S}_{\mathrm{A}}$ is the order parameter of the mesogens) and the worm-like concept used for semi-flexible polymers (where $\mathrm{S}_{\mathrm{B}}$ is the order parameter of the backbone), these authors described various nematic phases: a nematic phase of $N_{I I I}$ type where both $S_{A}$ and $S_{B}$ are positive and a $N_{I}$ type one where $S_{A}$ is positive and $S_{B}$ is negative. In this sense the "side-on" homopolymer $\left(\mathrm{PA}_{644}\right)$ corresponds to a nematic phase of $\mathrm{N}_{\text {III }}$ type while the "side-end" homopolymer ( $\mathrm{PA}_{61}$ ) is represented by a nematic phase of $N_{I}$ type. Renz and Warner [40] described the equilibria of such nematic phases. This model predicts that the decreasing of the order parameter $S_{B}$ of the backbone from a $N_{\text {III }}$ phase leads to a $N_{I}$ nematic phase (Ref. [40], Fig. 4) and assuming that $S_{B}$ is a function of the anisotropy ratio, this behavior is now experimentally verified via our side-on/side-end copolymer system.

\section{Conclusion}

Finally, it may be said that the aim of this work is reached with a series of new copolymers that show a systematic variation of the global chain anisotropy from a prolate-shape nematic regime to an oblate-shape one. As a consequence of their chemical structure the $\mathrm{S}_{\mathrm{A}}$ phase disappears even in copolymers containing a small proportion of side-on fixed groups. Correlatively the prolate shape of the chain conformation is reinforced in the nematic phase. 
Quantitatively, this evolution could be monitored selecting different kinds of mesogenic groups and/or different lengths of their corresponding spacers. In fact, measurements using new systems could provide more experimental evidence for a strong straightforward relation between the chain anisotropy and the nematic order in the prolate-shape nematic regime.

\section{References}

[1] Leube H.F. and Finkelmann H., Makromol. Chem. 191 (1990) 2707.

[2] Leube H.F. and Finkelmann H., Makromol. Chem. 192 (1991) 1314.

[3] Achard M.F., Lecommandoux S, and Hardouin F., Liq. Cryst. 19 (1995) 581.

[4] Pugh C., Liu H., Arehart S.V. and Narayanan R., Macromol. Symp. 98 (1995) 293.

[5] Takenaka S. and Yamasaki K., Mol. Cryst. Liq. Cryst. 258 (1995) 51.

[6] Hessel F. and Finkelmann H., Polym. Bull. 14 (1985) 375.

[7] Hessel F., Herr R. and Finkelmann H., Makromol. Chem. 188 (1987) 1597.

[8] Hessel F. and Finkelmann H., Makromol. Chem. 189 (1988) 2275.

[9] Zhou Qi-Feng, Li Hui-Min and Feng Xin-De, Macromolecules 20 (1987) 233.

[10] Zhou Qi-Feng, Li Hui-Min and Feng Xin-De, Mol. Cryst. Liq. Cryst. 155 (1988) 73.

[11] Keller P., Hardouin F., Mauzac M. and Achard M.F., Mol. Cryst. Liq. Cryst. 155 (1988) 171.

[12] Gray G.W., Hill J.S. and Lacey D., Angew. Chem. Int. Engl. Adv. Mater. 28 (1989) 1120.

[13] Hardouin F., Méry S., Achard M.F., Mauzac M. and Davidson P., Liq. Cryst. 8 (1990) 565.

[14] Lewthwaite R.W., Gray G.W. and Toyne K.J., J. Mater. Chem. 2 (1992) 119.

[15] Percec V. and Tomazos D., J. Mater. Chem. 3 (1993) 643.

[16] Pugh C. and Schrock R., Macromolecules 25 (1992) 6593.

[17] see for example: Finkelmann H. and Rehage G., Adv. Polym. Sci. 60-61 (Springer Verlag, Berlin, 1984) p. 99; Shibaev V.P. and Platé N.A., Adv. Polym. Sci. 60-61 (Springer Verlag, Berlin, 1984) p. 175; Mc Ardle, Side chain Liquid Crystal Polymers (Blackie, Chapman and Hall, New York, 1989).

[18] Hardouin F., Mery S., Achard M.F., Noirez L. and Keller P., J. Phys. II France 1 (1991) 511; and Erratum, J. Phys. II France 1 (1991) 871.

[19] Méry S., PhD Thesis 1990 Bordeaux, France.

[20] Hardouin F., Leroux N., Méry S. and Noirez L., J. Phys. II France 2 (1992) 271.

[21] Leroux N., Keller P., Achard M.F., Noirez L. and Hardouin F., J. Phys. II France 3 (1993) 1289.

[22] Leroux N., PhD Thesis 1992 Bordeaux, France.

[23] Leroux N., Mauzac M., Noirez L. and Hardouin F., Liq. Cryst. 16 (1994) 42.

[24] Lecommandoux S., Noirez L., Richard H. , Achard M.F., Strazielle C. and Hardouin F., J. Phys. II France 6 (1996) 225.

[25] Hardouin F., Leroux N., Noirez L., Keller P., Mauzac M. and Achard M.F., Mol. Cryst. Liq. Cryst. 254 (1994) 267.

[26] Noirez L., PhD Thesis, Université Paris XI, Orsay, France (in French) (1989).

[27] Noirez L., Keller P. and Cotton J.P., Liq.Cryst. 18 (1995) 129; and references therein.

[28] Lecommandoux S., Noirez L., Mauzac M. and Hardouin F., J. Phys. II France 4 (1994) 2249.

[29] Gray G. W., Hill J. S. and Lacey D., Adv. Mat. 28 (1989). 
[30] Achard M. F., Leroux N. and Hardouin F., Liq. Cryst. 10 (1991) 507.

[31] Noirez L., Cotton J.P., Hardouin F., Keller P., Moussa F., Pepy G. and Strazielle C., Macromolecules 21 (1988) 2891.

[32] Hardouin F., Méry S., Achard M.F., Mauzac M. and Davidson P., Liqq. Cryst. 8 (1990) 565.

[33] Cherodian A.S., Hughes N.J., Richardson R.M., Lee M.S.K. and Gray G.W., Liq. Cryst. 14 (1993) 1667.

[34] Diele S., Roth K. and Demus D., Cryst. Res. Technol. 21 (1986) 97.

[35] see for example: Noël C., Side chain liquid crystal polymers, C.B. Mc Ardle Ed., Chap. 6 and references therein (Blackie, 1989).

[36] a) Cotton J. P., Decker D., Benoît H., Farnoux B., Higgins J., Jannink G., Ober R., Picot C. and des Cloizeaux J., Macromolecules 7 (1974) 863; b) Cotton J. P., Neutron, X-Ray and Light Scattering, P. Linder, Th. Zemb Eds. (Elsevier, 1991).

[37] Boué F., Nierlich M. and Leibler L., Polymer 23 (1982) 29.

[38] Lapp A., Picot C. and Benoît H., Macromolecules 18 (1985) 2437.

[39] Wang X.J. and Warner M., J. Phys. A : Math. Gen. 20 (1987) 713.

[40] Renz W. and Warner M., Proc. R. Soc. Lond. A417 (1988) 213. 